\title{
The Inspiration of Transformation-The Creation and Aesthetic Appreciation of Ice Engraving
}

\author{
$\mathrm{Li} \mathrm{Yang}^{1 \mathrm{a}}$,Sun Hai Ping ${ }^{1 \mathrm{~b}}$ \\ ${ }^{1}$ Kunming University, China, Kunming 650214 \\ appigg@qq.com’bshp791004@163.com
}

Keywords: Ice engraved print; Innovation; Materials; Aesthetic

\begin{abstract}
Ice engraving is a special form of prints. Its medium is ice, while it is inherits traditional painting-creation techniques. Ice engravers are also boldly trying new concepts and methods of expression. Their selection of material has completely stepped out from the traditional ways, which enables it to break free from the restrictions of the environment. Factors such as temperature and time, give it very special characteristics, such as transience, contingency, uniqueness and innovation, which also vividly reflect the regional characteristics of culture and the artistic innovative spirit. The creative process and aesthetic characteristics of ice engravings are valuable for their research significance. This article briefly analyzes and expounds views on these issues...
\end{abstract}

\section{Introduction}

Ice engraving is the youngest and the most characteristic form in the engraved print family. Its motherboard is ice. The artists make the mold of their paintings on the surface of ice by using knives, shovels, and other auxiliary tools, then monotype the paintings using the Chinese art paper. The particularity of the creation form makes it break through the identity of stylized, repetitive form associated with the Chinese traditional colored woodcut. Namely, it inherits the characteristics of traditional print, and bears all the advantages of prints such as woodcuts, lithographs and copperplate etching, yet its expressions are much richer. We can not deny that this is a huge creative breakthrough in terms of texture, techniques, and lasting appeal. The selection of material has completely stepped out from the traditional ways. The restrictions of material and environment, and factors such as temperature and time, give it very special characteristics such as transience, contingency, uniqueness and innovation, which reflect the regional characteristics of culture and the artistic innovation spirit. The creative process and aesthetic characteristics of ice engravings are valuable for their research significance. All these make it an offbeat version of engraved prints, the wonderful flower of ice sculpture.

\section{The Extension of the Art Forms with Water Media}

In Chinese, there is an idiom: "Complete pure water is beneficial to everything." that is to say "The goodness of wisdom is like water, beneficial to everything." Chinese traditional painting and calligraphy arts all used water as a medium. When the natural flow of water and the essence from the artist mix together, they create the great spiritual power of artistic works. Water is an important medium and element of visual arts, and the Chinese guidance and control of water is a unique expression of Chinese philosophy.

Ice is water but is colder than water.

Ice is also the solid form of water. The fluidity and solubility of water make it more manageable when it becomes ice. The image of the water changes along with the change of external factors, as it does not have a fixed shape. This is exemplified by yet another Chinese idiom which describes the fluidity of water so: "In a hollow, it fills the void; in the mountains, it fills the valleys." Yet the plasticity and mold of ice is different from water. Ice can be cut, sculpted, pasted and carved. It has both the plasticity of the wood and the stiffness of the slate, and it is also 
transparent and easy to break.

So, ice engraving print is another form and model of water-medium art. It is the extension of the water medium visual arts. It belongs to both the category of ice sculpture and also print, and it has the characteristics and aesthetic taste of both. It is an alternative form and extremely rare in visual arts.

In the sculpture method, "water" dissolves the color ink, and it is sculpted by pen. Its characteristics are shading, permeating and deposition. The carving method has the flavor of both print art and ice sculpture art, yet its unique carving method gives it a certain rough, almost child-like result. In the process of creation, ice engraving print is fortuitous because the technique is very difficult and can't be replicated exactly, and it belongs to the family of naturally destroyed print, so we called it "Out of print ice engraving". Although ice engraving print came into being for a very short time, it has great value and status in the history of the Chinese print, and brings the new aesthetic ideas associated with the ice sculpture art.

The fortuity of the ice engraving creation makes the artists pay more attention to "arousing" inspiration and the creative power which is very important in the creating process. The carving method of ice engraving is thick, solid, rough, bold, uncompromising attention to detail, ingenious and meticulous, both crude and artful, but it has the regional identity from the north of China, and also it has taken on natural texture from the nature. No matter what color, the black and white prints or the chromatic prints, they both have a sweet and desolate beauty.

\section{The Living Spirit of the Artist's Inspiration}

The artist's inspiration is a very important factor of ice engraving. As the creator of ice engraving art, Zhu Xiao Dong continues to inspire new practices. While others created only monochromatic ice engraving, he tried color ice engraving. Because of the similarity between the color pigment painted on ice and on glass, the pigment isn't easy to attach, control, or evenly distribute. This can cause messy situations like the painting turning out very dim. But the artist unremitting pursuit and spiritual gifts make inspiration flow out then the art becomes a classic art form. Zhu Xiao Dong repeated the experiment, again and again, until he found the proper concentration and dilution of pigment, and the way of time controlling the application. He searches the ice engraving staining techniques, and then promotes the development of ice engraving. Ice engraving inherits the characteristics of the traditional print just like strong contrast, concise composition, lively line, and having the effect of ink painting. However the special engraving techniques and media of ice sculpture art showcase its own creative charm of multi-discipline comprehensive visual arts.

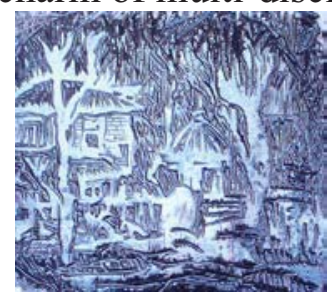

Figure 1: Old building in Harbin (Ice engraving) Source: Zhu Xiaodong,2013

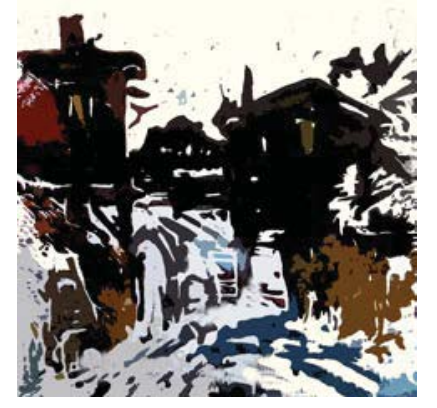

Figure 2: Old building in Harbin 1 


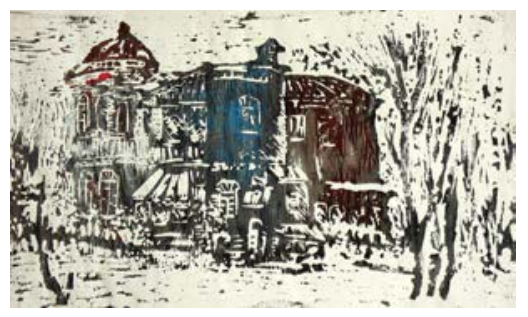

Figure 3: Old building in Harbin 2(Print of ice engraving)

Yao Zui said, "Nature follows the ancient way, but the exterior shows the feature nowadays," "changeable and convenient." That means in the different period and background, the artistic content, expression and form is combined into something different. That is innovative but also has inherited the innovations of predecessor. The creation and development of ice engraving breaks the consensus of material of the inherent prints, never to follow the old way, and makes a new bold breakthrough. From the perspective of the creative practice, ice engraving emphasizes the originality and creativity in the art creation. Once the art loses its creation then it loses its vitality. The monk Dan Dang said in his poem, "Time destroys beauty, won't last through the next year spring. Although Xi Shi has an alluring glow to her skin, she is often worn down by a frown." Here it refers the issues of artistic creation and innovation, which euphemistically points out the importance of artistic creative originality. Ingenuity is one of the necessary premises of art development. Creation is a motivating force of such development; scholasticism often leads to the narrower and narrower road.

Schiller said, "Poet is nature, or they seek nature. Under the former emotion, he is a simple poet, in the latter case, he is a sentimental poet." "Simplicity" and "sadness", these two concepts can be extended to the aesthetic style of art or the aesthetic appreciation form. Ice engraving art expression contains two moods, sadness and Simplicity. Sad feelings can be an important motivation of the artist's emotional expressions and themes. And the simple element of simplicity is the result of the artist's wandering thoughts. Art can light up the future of life, and can guide people out of confusion. This pioneering creation comes from the instinct of artists' pursuit of the life spirit, the art of questioning practice experience, ceaseless artistic thinking; and the sincere essence of bold attempt and not being afraid of failure.

Picasso said, "I could already paint like Master Raphael when I was a little boy, but I have to spend my whole lifetime to learn how to paint like a little child." So, it is because of the practice in his life, he found "the naive" is rare and hard to find. Allegedly it is easy when it goes from simple to complex, yet it is hard to go from complex to simple. This is an uncomfortable contradiction: time can't be stopped, and naivety disappears gradually in the process of the increasing cognizance--if you want to retrieve your naivety, it is not easy!

In the creation of ice engraving print, the artists obviously show the wisdom and artistic attainment that we called "your suitable naivety," which includes the "naive" creative style and aesthetic experience. Its own artistic inspiration and life spirit make the ice engraving a very uncommon aesthetic value. The natural and uniqueness of the material of ice engraving print take the aesthetic meaning of ice engraving back to the wonderful place we called "retrieve to simple", a great significant environmental state.

\section{The Beauty of the Special Material}

Chinese aesthetics uses concepts such as dry, pale, thin, cold, lonely, decadent, and sparse, which reflect the awareness of the natural universe. Chinese art attaches great importance to the experience of enduring hardship, such as a lifetime spend roving in the wind and rain. "Cold and concise" is the symbol of this life spirit. "Pure and noble" is also the generalization of the unique temperament of ice.

As opposed to "soft and gorgeous," "cold and concise" contains the experience of the calm state of mind, it keeps the indomitable spirit and the prayer for survival from adversity. The phrase 
"cold and concise" evokes a beautiful mood like "words escape me" and "lost for words", something both miserable and beautiful, like the depression of darkness before dawn and the expectation of life. At this time it is the complex feeling of silent beauty, the possibility of owning everything, and in a curious position between rationality and sensibility. Coldness and comeliness, loneliness and degradation, the years are gone and never return, and life is short. All of these, in painting aesthetics, represent a kind of elegance, a kind of philosophy, a kind of mental state, and a way of life. The condensation state of ice itself may cause the phrase "cold and chill" or "simple and remote" to come to mind. Artists empathize with ice, wander in painting, draw and sculpt on the ice, graving and transparent, glittering and bright. From the vast vehemence that hides in the cold of ice, the ice artist can coax flourishing beauty. That is the visual and visceral contradiction of ice engraving that people find so intriguing.

Ice, as a material, confronts with and melts into the human emotions, only the "simple and elegant" can have the pleasure of "tranquility and lighting." Ice engraving acquires a certain vitality from these fantastic and wonderful feelings associated with its media. The artist's emotional catharsis also imbues it with a certain artistic spirituality. Snow and ice sculpture is one of the most special kinds of expressions of art. In the unique environment and comprehensive creation experience, the beauty of the material itself raises the art form. Also the natural limitations of ice that make ice engraving templates difficult to keep, which means only limited pieces of ice works remain. Thus, the material beauty of ice engraving is unique from other engraving materials. Between creation and loss, strong aesthetic expectation and artistic carriers of disappearing work, the artist and the audience will produce more than just visual aesthetics even creating a spiritual and sensory shock.

\section{Summary}

In a word, ice engraving is free from the vulgarity of the complication of community life. It is beyond the basic definition of prints, using the material transformation of ice sculpture to ushers in new life in the form of art. It is both the gift of nature, and the result of people to grasping to understand and appreciate that natural beauty. The beauty of ice engraving is from the material itself and the expression of the artist's feelings. The beauty is in nature, in the experience, in silence, in the glittering and bright material, in the ink marks, in the drops of condensation, in the moments' eternity.

\section{ABOUT THE AUTHORS}

Li Yang: Li Yang is a Lecturer in Fine Arts at Kunming University in Kunming, Yunnan, China. She earned her M.A. in Chinese Minorities' Art from Kunming University of Science and Technology in 2011. She earned her B.A. in Landscape Design from Southwest Agriculture University in Chongqing, China, in 2004. She has research interests in landscape design, Chinese minorities' landscapes, and art teaching theory.

Sun Hai Ping: Sun Hai Ping is an associate professor in School of Fine Art and Design of Kunming University in Kunming, Yunnan, China. He earned his M.A. in Fine Art from Yunnan Normal University in 2012. He earned his B.A. in Fine Art from Harbin Normal University in Harbin, Heilongjiang, China, in 2004. He has research interests in fine art theory, ice and snow art, and lacquer art.

\section{REFERENCES}

[1]Friedrich Von Schiller. Translated by Jiang Kong-Yang. 1979.Simple poetry and sentimental poetry .Shanghai: Shanghai Publishing House

[2]Volk, Gregory. 2000. “Art on Ice” Art in America. 0004-3214,20000901 
[3]Zhu Liangzhi.2006.The fifteen lectures of Chinese aesthetics.Beijing:Beijing University Publishing House. 730110586X

[4]Zhu Liangzhi.2006. The life's spirit of Chinese art. Hefei:Anhui Education Publishing House. 7533616901

[5]Peng Jixiang.2006. The Contemplation on Art. Beijing:Beijing University Publishing House. 7301107102 